ARTICLE

\title{
In situ cryo-electron tomography reveals gradient organization of ribosome biogenesis in intact nucleoli
}

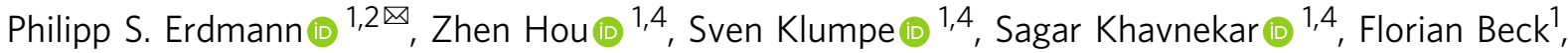 \\ Florian Wilfling (1) ${ }^{1,3}$, Jürgen M. Plitzko (i) ${ }^{1} \&$ Wolfgang Baumeister (D) ${ }^{1 凶}$
}

Ribosomes comprise a large (LSU) and a small subunit (SSU) which are synthesized independently in the nucleolus before being exported into the cytoplasm, where they assemble into functional ribosomes. Individual maturation steps have been analyzed in detail using biochemical methods, light microscopy and conventional electron microscopy (EM). In recent years, single particle analysis (SPA) has yielded molecular resolution structures of several pre-ribosomal intermediates. It falls short, however, of revealing the spatiotemporal sequence of ribosome biogenesis in the cellular context. Here, we present our study on native nucleoli in Chlamydomonas reinhardtii, in which we follow the formation of LSU and SSU precursors by in situ cryo-electron tomography (cryo-ET) and subtomogram averaging (STA). By combining both positional and molecular data, we reveal gradients of ribosome maturation within the granular component (GC), offering a new perspective on how the liquid-liquid-phase separation of the nucleolus supports ribosome biogenesis.

\footnotetext{
${ }^{1}$ Max Planck Institute of Biochemistry, Martinsried, Germany. ${ }^{2}$ Fondazione Human Technopole, Milano, Italy. ${ }^{3}$ Max Planck Institute of Biophysics, Frankfurt, Germany. ${ }^{4}$ These authors contributed equally: Zhen Hou, Sven Klumpe, Sagar Khavnekar. ${ }^{凶}$ email: erdmann@biochem.mpg.de; baumeist@biochem.mpg.de
} 
T he assembly of eukaryotic ribosomes is a complex process involving up to 200 proteins and RNAs ${ }^{1}$, which are transcribed from a single copy of ribosomal DNA (rDNA). Purified and spread rDNA (Miller spreads) have been used to investigate the nuclear stages of pre-ribosome formation in vitro. This has enabled the structural identification of both the active polymerase (Pol I) as well as "terminal knobs", which have been suggested to correspond to SSU and LSU precursors ${ }^{2-4}$. In cells, these early steps of ribosome biogenesis take place in a dedicated nuclear compartment, the nucleolus, which has been studied extensively using fluorescence and conventional plasticembedded electron microscopy $(\mathrm{EM})^{5}$. Since nucleoli are not held together by a membrane, their isolation, although feasible ${ }^{6}$, poses a problem for studying their natural organization by cryoelectron microscopy (cryo-EM), which promises much improved structural preservation and resolution compared to conventional EM.

Several key aspects of nuclear ribosome biogenesis have recently been elucidated by high resolution SPA of various preribosomal particles in different species ${ }^{7-16}$. While these atomic models have provided valuable insights, they cannot capture the spatiotemporal organization of ribosome maturation. Moreover, the need to stabilize and enrich intermediates via genetic or biochemical manipulation, could artificially favor certain states over others.

To overcome these limitations and to reconcile the wealth of morphological and molecular information available, we turned to in situ cryo-ET ${ }^{17}$ and visual proteomics ${ }^{18}$ to investigate the native organization of the nucleolus and its role in ribosome biogenesis.

\section{Results and discussion}

Cryo-focused ion beam (FIB) milling allows preparation of electron-transparent samples preserved in their native state by cryo-fixation without the need for chemical agents and without the artifacts known from cryo-sectioning ${ }^{19,20}$. Traditionally, ribosome biogenesis has been investigated in the yeast Saccharomyces cerevisiae (S. cerevisiae) because of its robustness and the availability of genetic tools ${ }^{21}$. However, the localization of $S$. cerevisiae nucleoli can be technically challenging in a pure cryogenic workflow due to their unpredictable cellular location ${ }^{22,23}$. We therefore chose the green alga Chlamydomonas reinhardtii (C. reinhardtii) as it has an almost deterministic cellular architecture, eliminating the need for correlative targeting ${ }^{24}$. Although Chlamydomonas might have a simpler ribosome assembly pathway than other eukaryotes ${ }^{25}$, many biogenesis factors are conserved. Moreover, C. reinhardtii shows all the hallmark features common to nucleoli: At its center, the fibrillar and dense fibrillar components (FC and DFC), site of rDNA transcription, can be identified. Both are surrounded by the granular component (GC), where pre-ribosome assembly is thought to take place.

To resolve their nucleolar organization, we subjected Chlamydomonas to our cryo-FIB workflow and obtained a large number of suitable tomograms slicing individual nucleoli at various angles and heights (Supplementary Fig. 1). Overall, the data shows the expected tripartite organization (Supplementary Fig. 1J, K). Additionally, owing to their native preservation, individual particles can now be distinguished. While most of the larger complexes appear to be confined to the nucleolus (Fig. 1A, white arrow), a fact also reported by conventional $\mathrm{EM}^{26}$, a small number of large, electron-dense particles is found in the nucleoplasm (Fig. 1A, black arrows). Beyond this qualitative assessment, template matching was used to search for and identify specific targets within the $3 \mathrm{D}$ data ${ }^{27,28}$. Using an unstructured reference (Supplementary Fig. 2), the pre-60S and SSU processome were found as the two dominant types of nucleolar complexes (Fig. 1B, C, Supplementary Movie 1). While the small subunit precursor seems mostly restricted to the nucleolus, some LSU-like complexes are also detected far out in the nucleoplasm (Fig. 1B), which is consistent with their expected remodeling prior to export to the cytosol ${ }^{15,16,29}$.

In tomograms that covered a sufficiently large area, we observed a distinct orbital pattern of pre-ribosomal particles around the nucleolus (Supplementary Fig. 2). We therefore devised an algorithm that fits spheres to their combined 3D positions to determine the size and centers of the nucleoli (Fig. 1D and Supplementary Fig. 3). At $599 \pm 29 \mathrm{~nm}$ (mean \pm SEM; $n=26$ tomograms), their mean radius agrees with previous studies ${ }^{30}$. The individual probability distribution functions (PDFs), however, reveal distinct localization patterns of pre-60S and SSUprocessome complexes (Fig. 1E). While both show an increase in particle number with distance from the center, the SSU precursor has a significantly sharper peak around the calculated mean radius - hereafter referred to as the "surface" of the nucleolus. This gradient architecture is intriguing because the particles seem to delineate the very transition from the GC to the nucleoplasm. While numerous studies have attempted to assign functional roles to individual nucleolar components and to localize specific ribosomal precursors within them, it is still unclear whether the layered $\mathrm{FC} / \mathrm{GC}$ organization is required for ribosome formation or rather a consequence thereof. Nevertheless, there is evidence that formation of the rRNA transcripts is key to the formation and maintenance of nucleolar organization ${ }^{31}$. Unlike other organelles, nucleoli are not surrounded by a membrane and arise from liquidliquid phase separation (LLPS). Recent studies have shown that LLPS is responsible for the assembly of the dense fibrillar component in the human nucleolus ${ }^{32}$, and that the FC-GC core-shell architecture of Xenopus laevis oocytes, is based on the surface tension of the individual protein component ${ }^{33}$. Although heterotypic multicomponent interactions have been suggested to drive cellular liquid-liquid phase separation ${ }^{34}$, it remains unclear how such an organization is maintained on a per-particle basis.

Given the differential localization of LSU and SSU precursors, we decided to further explore the nucleolar architecture in Chlamydomonas. While numerous smaller particles can be observed toward the center of the nucleoli (Fig. 1A and Supplementary Fig. 1), template matching with unstructured references of different sizes did not yield any additional well-defined averages. This might be due to the dynamic nature of preribosomes and certain experiment- and sample-related limitations, e.g. particle number, pixel size, and SNR. In combination, these factors might preclude robust classification and thereby identification of smaller particles such as the pre-40S.

To overcome these limitations of STA, we turned to an approach based solely on image analysis. Densities within each tomogram were segmented and their average size was determined after denoising and filtering (See Methods for details). With the nucleolar centers as reference points, we performed a distance analysis, finding that, on average, the apparent particle mass increases throughout the granular component, with an initial maximum at the surface of the nucleolus where most pre-60S and SSU processomes are detected (Fig. 2A, top).

To further corroborate this finding, we performed an analysis of the particle density as a function of mass and distance from the nucleolar center. This revealed two major properties: an almost constant background of smaller particles up to $\sim 2.5 \mathrm{MDa}$, and a spike of larger complexes (3-5.5 MDa) toward the surface of the nucleolus (Fig. 2B; dashed line). This suggests that GC particles, which at this point lack an exact identity, are partitioned by size and thus potentially by developmental stage.

We therefore hypothesized, that this might also be true for the later stages, where averages can be obtained and thus identities can be assigned. To test this idea, we performed additional rounds 


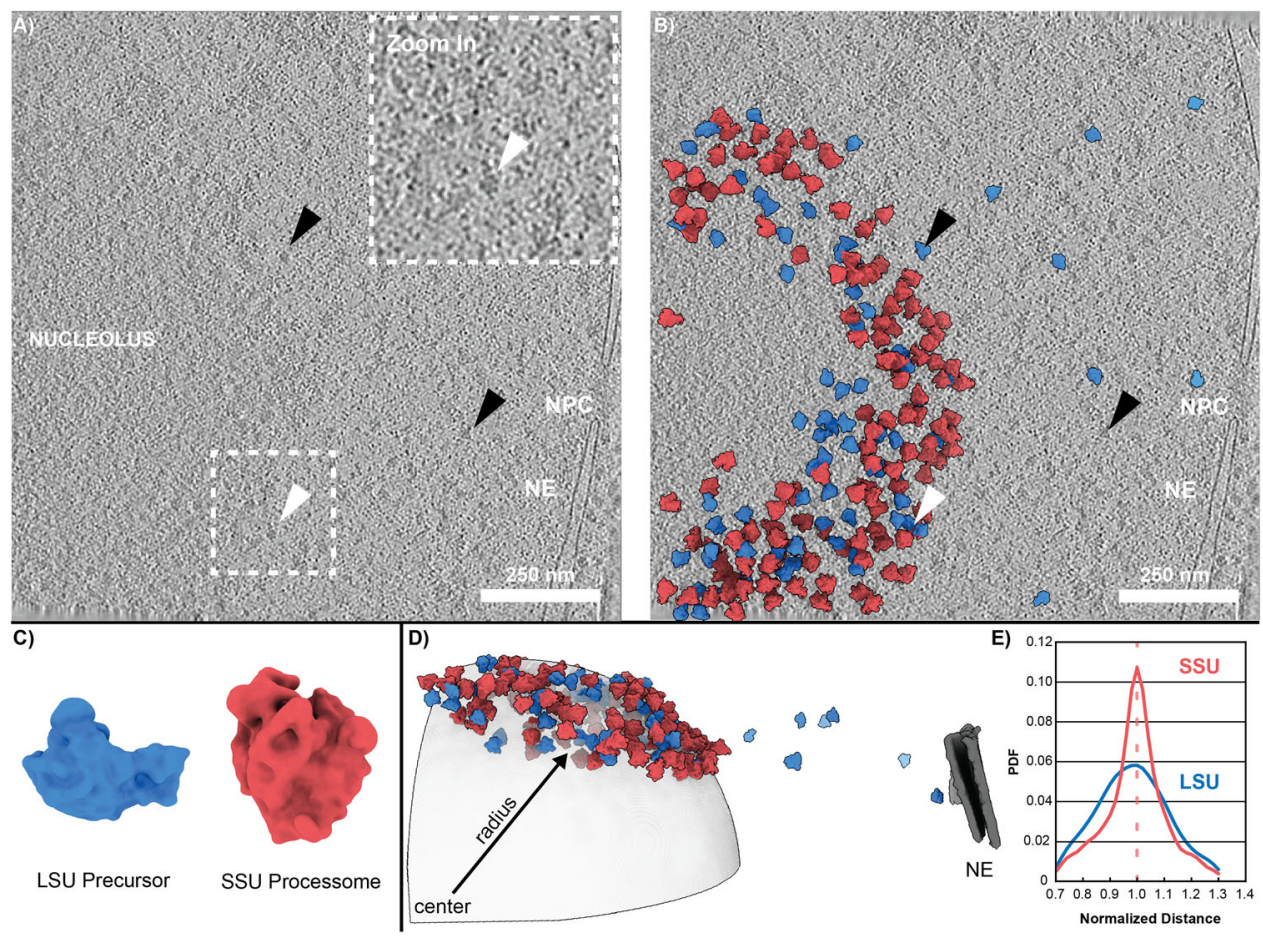

Fig. 1 Structural and molecular organization of the C. reinhardtii nucleolus. A The C. reinhardtii nucleolus is clearly visible as a dense structure decorated with large particles (white arrow \& zoom in). Some of these particles (black arrows) are also found floating in the nucleoplasm. B, C Using reference-free template matching, pre-60S particles (blue) and SSU processomes (red) are detected ( $n=85$ tomograms). D Their 3D organization is consistent with a sphere decorated by pre-ribosomes ( $n=26$ tomograms for which spheres could be fit). E The small subunit precursors show a characteristically different probability density function (PDF) than the pre-60S, with a sharper peak around the nucleolar surface $(n=26$ tomograms; Fisher-Pitman permutation test ${ }^{60}$ with significantly smaller variance for the SSU: $p=1.0 \mathrm{E}-06$ ). LSU Large Subunit, SSU Small Subunit, NE Nuclear envelope, NPC Nuclear Pore Complex. Source data are provided as a Source Data file.
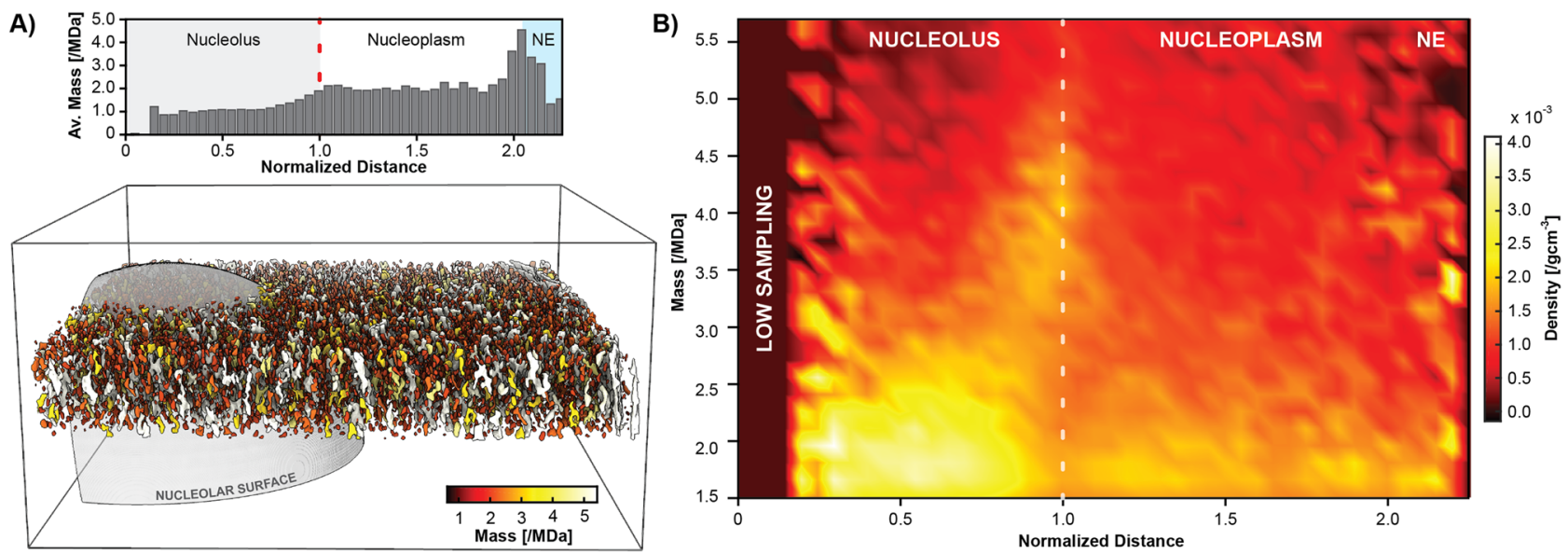

Fig. 2 Image-based analysis of the nucleolar organization. A Individual tomograms can be denoised ${ }^{50}$ and filtered to allow segmentation of nuclear particles (bottom). Analysis of their apparent mass (top) shows an increase in apparent mass per particle toward the surface of the nucleolus starting at $\sim 0.75$ of the normalized distance. Before and after that, the average mass remains relatively constant until the nuclear envelope is reached. B Spectral analysis of particle mass vs. normalized distance over all tomograms with sphere fits shows two main features: 1. There are constantly high levels of particles up to a size of $\sim 2.5 \mathrm{MDa}$, except around the surface of the nucleoli (dashed line). 2 . At this distance, a lack of smaller masses is compensated by a burst of large complexes, consistent with the detection of pre-ribosomal particles such as the SSU processome and the pre-60S. NE Nuclear envelope. Source data are provided as a Source Data file.

of template matching, this time using the previously obtained, well-structured averages as starting points. In order to avoid reference bias, a template-truncation strategy was implemented ${ }^{35}$, since otherwise false positive rates can be inflated (Supplementary Fig. 4A). Based on the new global averages, sub-classification (Supplementary Fig. 4B) was performed for both precursors. For the pre-60S, we found three major classes that resemble late nucleolar and nucleoplasmic intermediates from published SPA studies ${ }^{12}$, which validates our classification approach (Supplementary Fig. 8A-C). For the small subunit processome, there has been little information on conformational heterogeneity until recently when additional intermediates were described using SPA 

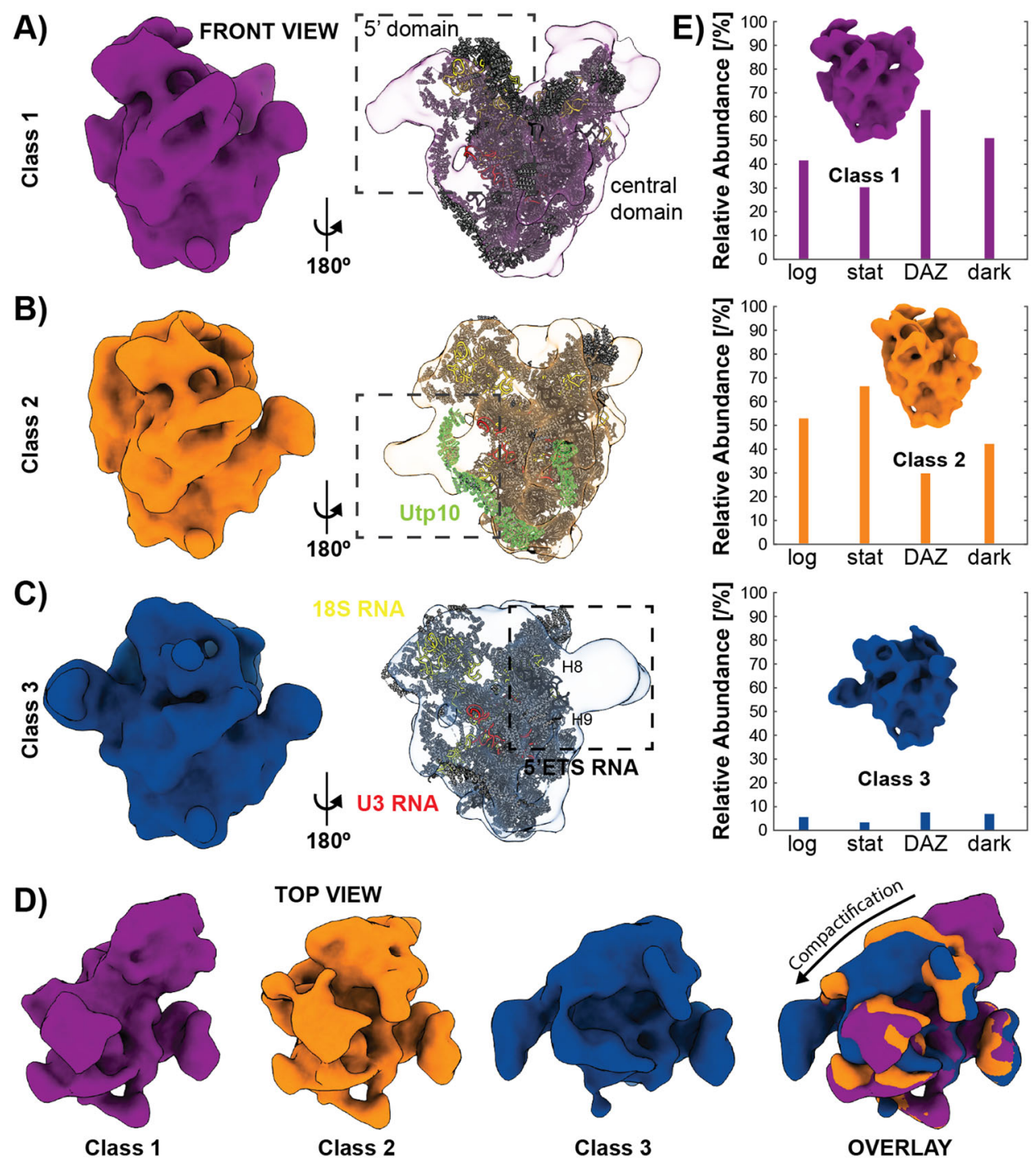

Fig. 3 Structural heterogeneity of in situ SSU processome particles. A-C Three distinct SSU processome class averages (Class 1-3) with different similarity to published structures are detected. D There is a clear trend from Class 1 to Class 3 toward a more compact arrangement of the $5^{\prime}$ and central domains. This is particularly evident when all three STA structures are aligned and superimposed, and indicates a sequence of maturation (Supplementary Movie 2). E The relative abundance of each class is subject to external stimuli. While Class 2 dominates during logarithmic (log) and stationary growth (stat), Class 1 becomes the predominant form when treated with diazaborine (DAZ), a drg1 AAA-ATPase inhibitor, and when ribogenesis is stimulated (dark). PDB model for A-C: 5WYJ. See Supplementary Fig. 6 for a structural comparison with other maps. Source data are provided as a Source Data file.

and refined isolation and preparation techniques 36,37 . Since our in situ approach does not require any biochemical treatments, it provides an unbiased view of all intermediates in their native context. After implementing a rigorous classification strategy and checking for cross-effects (Supplementary Fig. 5), we found three distinct SSU processome classes (Fig. 3A-C). Despite their moderate resolution $(\sim 25 \AA)$, large domains and their movement between states can still be interpreted and compared to single particle results. Overall, our in situ structures are in good agreement with published SPA maps (Supplementary Fig. $6 \mathrm{~A}-\mathrm{C})^{8,10,38}$, including iconic features such as helix 44 and the base region of UtpA, which is formed by the beta-propellers of Utp17 and Utp $8^{38}$. However, unlike the single particle structures, some densities seem to be more continuous (Supplementary Movie 2) and there are features which are not present in the SPA maps. While these certainly could represent Chlamydomonas-specific subunits, they could also correspond to cofactors lost during purification for single particle cryo-EM.
Some of the observed variance could be due to both lower resolution and species-specific subunits. However, the small changes reported so far between organisms that are evolutionarily quite distant is more indicative of physiologically relevant structural features ${ }^{8,38}$. Of the three, SSU processome Class 2 shows the highest similarity to published maps overall (Supplementary. Fig. 6D), except for an additional density found around the location of UTP10 (Fig. 3B). Contrarily, Classes 1 and 3 appear significantly different and hence merit further investigation.

Compared to the available SPA-structures, Class 1 with its $5^{\prime}$ domain distinctly shifted away from the central domain (Fig. 3A) displays a more open conformation than published SSU processome intermediates. It is therefore reasonable to assume that Class 1 represents an early state. In contrast, Class 3, the least abundant class, is fairly similar to published structures with respect to both orientation and location of the $5^{\prime}$ domain, albeit slightly more compact. Additionally, there is a prominent density next to Utp14 at helix 8 and 9 within the 5'ETS RNA, the 
suggested binding site of MTR4 and by proxy the nuclear exosome (Fig. 3C). Comparing all three maps, there is an apparent compactification from Class 1-3, during which the $5^{\prime}$ moves closer to the central domain and therefore its mature state (Fig. 3D). Finally, comparing our structures to SSU intermediates recently solved by SPA (Supplementary Fig. $6 \mathrm{E}$ ) ${ }^{36,37}$ shows that the order of Class 1-3 is consistent with progressing stages of maturation. Class 1 even seems to pre-date the earliest isolated structures so far and Class 3 corresponds to a late, exosomebound intermediate (Supplementary Fig. 6F, G).

To further substantiate this proposed order, we examined the relative abundance of SSU intermediates in Chlamydomonas cells that were subjected to different stimuli (Fig. 3E). During log phase, SSU Class 1 and 2 are detected at $42 \%$ and 53\%, respectively, while Class 3 makes up only $5 \%$, potentially suggesting a short-lived intermediate. Under stationary growth conditions when nutrients are limited and consequently the rate of ribosome biogenesis is low, Class 2 becomes the predominant form by far (66\%). Interestingly, this also matches experimental conditions of earlier SPA studies and hence might explain the strongest similarity of Class 2 with these maps (Supplementary Fig. 6D). An opposite shift in abundance is observed when cells are treated with diazaborine (DAZ), a drug known to interfere with late-stage pre-60S biogenesis. DAZ inhibits the AAA-ATPase drg1 and in turn prevents the release and recycling of Rlp24 after shuttling the LSU precursor to the cytoplasm ${ }^{39,40}$. Brief DAZ-treatment of $C$. reinhardtii cells shifts both intermediates of the large and the small subunit toward the earlier states. While diazaborine has been reported to selectively affect the pre- $60 \mathrm{~S}^{39}$, which we also capture in the classification of our LSU precursors (Supplementary Fig. 7F), it is conceivable that the failure to recover important late-stage nucleolar LSU factors could also lead to changes in the biogenesis of the SSU processome. Alternatively, a lack of functional cytosolic $60 \mathrm{~S}$ subunits could initially stimulate ribosome biogenesis and hence result in an increase of early intermediates. As ribosome formation in Chlamydomonas is linked to its day/ night cycle, we prepared synchronized cultures and harvested the cells $4 \mathrm{~h}$ after dark phase initiation, the expected peak of ribogenesis ${ }^{41}$. This physiological stimulus again shifts the prevalence in favor of Class 1, making it the major SSU precursor at $52 \%$, followed by Class 2 at $42 \%$, and Class 3 at $6 \%$.

Having confirmed that SSU Classes 1-3 represent snapshots of progressive maturation, we checked for signs of spatial segregation, which would support our prior notion of a gradient organization within the GC. Focusing on the later stages, where STA averages can be obtained, we compared the normalized nucleolar center-to-particle distance of each individual complex, revealing a statistically significant increase in the mean from SSU Class 1 to 2 , and 3 (Fig. 4A). To investigate this distribution in more detail, we plotted the probability density function for each class, exposing gradients with individual peaks for each intermediate (Fig. 4B, C). Combined with the previous results, our analysis therefore supports the idea of progressive maturation of preribosomes within the granular component. In addition, the two peaks for SSU Class 3 PDF (Fig. 4B) may indicate alternate routes of exosome-binding and hence pre-rRNA processing, as has been proposed for several eukaryotic species ${ }^{42}$.

How do these findings fit into the larger picture of nucleolar organization, ribosome biogenesis, and LLPS? Our data suggest a model, in which starting from the center of the nucleolus, preribosomal particles are processed successively, advancing both in size and maturation as they move radially outward until structurally welldefined intermediates appear. Furthermore, we show that the small subunit precursor in particular acts as a marker that defines the transition from the nucleolus to the nucleoplasm and hence from a liquid-liquid phase separated to a "normal" solution state. At this boundary, the SSU processome, which merely acts as a construction scaffold for the pre- $40 \mathrm{~S}$ particle, falls apart and is therefore not found much further beyond this point (Fig. 4D). Our data provides the first molecular-level evidence for theoretical work, which predict that the liquid-liquid phase separation organization of the nucleolus is a result of concentration gradients and the multivalent interactions of the pre-ribosomal complexes. As particles mature, it is suggested that sites for processing and thus for interaction with cofactors localized to the nucleolus are progressively lost. Accordingly, the multivalence of interactions between pre-ribosomes and the LLPS environment decrease. A maturation gradient is therefore expected before particles are eventually driven out of the liquid phase-separated domain ${ }^{34}$. This idea is clearly supported by our analysis of particle size and molecular identity of the SSU processome and the pre-60S, providing evidence for the partitioning of key processing steps within the nucleolus. Moreover, the distinct orbital pattern, which results from the final stages of nucleolar pre-ribosome assembly, shows that LLPSlike organelles, despite lacking membranes, can still coordinate and thus control biochemical reactions at interfaces similar to their "classical" counterparts. Controlling the dissociation of the particles from the LLPS could furthermore represent an elegant way to synchronize ribosome biogenesis. If the release "signal" is an intrinsic characteristic of the intermediates, this could serve as an integrated form of quality control. Clearly, larger datasets are required to decipher the finer details of this sub-nucleolar organization. This includes analysis in other organisms, identification of the smaller GC particles that have not yet been identified, and localization of other intermediates suggested by SPA studies like the $5^{\prime} \mathrm{ETS}^{14}$. Nevertheless, our current results already show that the nucleolus creates an environment which helps to separate crucial events of ribosome biogenesis both in space and time.

\section{Methods}

Cell culture. Cells used in this study were Chlamydomonas reinhardtii strain mat34 (CC3994) acquired from the Chlamydomonas Resource Center, University Minnesota, MN, which produces a smaller growth phenotype ${ }^{43}$. Unless otherwise stated, cells were agitated at $100 \mathrm{rpm}$.

Log phase. From a plate culture, a liquid culture was inoculated in $40 \mathrm{~mL}$ Trisacetate phosphate (TAP) media $\left(0.02 \mathrm{M}\right.$ Tris base, TAP salts $\left(7.00 \mathrm{mM} \mathrm{NH}_{4} \mathrm{Cl}\right.$, $\left.0.83 \mathrm{mM}, \mathrm{MgSO}_{4}, 0.45 \mathrm{mM} \mathrm{CaCl}_{2}\right)$, Phosphate buffer $\left(1.65 \mathrm{mM} \mathrm{K}_{2} \mathrm{HPO}_{4}, 1.05 \mathrm{mM}\right.$ $\mathrm{KH}_{2} \mathrm{PO}_{4}$ ), Hunter trace elements $\left(0.134 \mathrm{mM} \mathrm{Na}{ }_{2}\right.$ EDTA, $0.136 \mathrm{mM} \mathrm{ZnSO}_{4}$, $0.184 \mathrm{mM} \mathrm{H}_{3} \mathrm{BO}_{4}, 40 \mu \mathrm{M} \mathrm{MnCl}_{2}, 32.9 \mu \mathrm{M} \mathrm{FeSO}_{4}, 12.3 \mu \mathrm{M} \mathrm{CoCl}_{2}, 10.0 \mu \mathrm{M} \mathrm{CuSO}_{4}$ $4.44 \mu \mathrm{M}\left(\mathrm{NH}_{4}\right)_{6} \mathrm{MoO}_{3}, 17.5 \mathrm{mM}$ acetic acid)). Cells were constantly illuminated $\left(\sim 90 \mu \mathrm{mol}\right.$ photons $\left./ \mathrm{m}^{2} \mathrm{~s}\right)$, aerated with normal atmosphere, and harvested $48-72 \mathrm{~h}$ after inoculation.

Stationary phase. A log phase culture was allowed to continue growing for seven days without dilution.

DAZ treatment. Log phase cells were treated with diazaborine (DAZ, $370 \mu \mathrm{M}$, Merck) for $2 \mathrm{~h}$ before plunge freezing.

Synchronization experiments. From a log-phase culture $(24 \mathrm{~h})$, a sample was taken and transferred to $40 \mathrm{~mL}$ of fresh TAP media. Cells were subsequently cultured in an isolated grow box with electrically controlled illumination using a $12 \mathrm{~h} / 12 \mathrm{~h}$ day/ night cycle. Every 2-3 days, the cell density was adjusted during the day period to $\sim 1000$ cells $/ \mu \mathrm{L}$ by manually diluting the sample with fresh TAP and removing excess media and cells. This was maintained for three such cycles. Finally, cells were harvested after $4 \mathrm{~h}$ of initiating the dark cycle.

Plunge freezing. A total of $4 \mu \mathrm{L}$ of cell suspension (1500-2000 cells/ $\mu \mathrm{L})$ were applied to holey carbon R2/1 copper, or holey $\mathrm{SiO}_{2} \mathrm{R} 1 / 4$ TEM grids (Quantifoil) and cells were plunge frozen on a Vitrobot Mark IV (FEI, settings: blotforce $=10$ blottime $=10 \mathrm{~s}$, temperature $=30^{\circ} \mathrm{C}$, humidity $=90 \%$ ) using back-side blotting only and using an ethane/propane bath. Samples were stored under liquid nitrogen until use.

FIB milling. Lamellas were prepared on a dual beam focused ion beam microscope (Quanta 3D FEG, FEI) equipped with a Quorum PP3000T cryo-system (Quorum Technologies, Laughton, United Kingdom) and a homemade $360^{\circ}$ cryo-stage 
A)

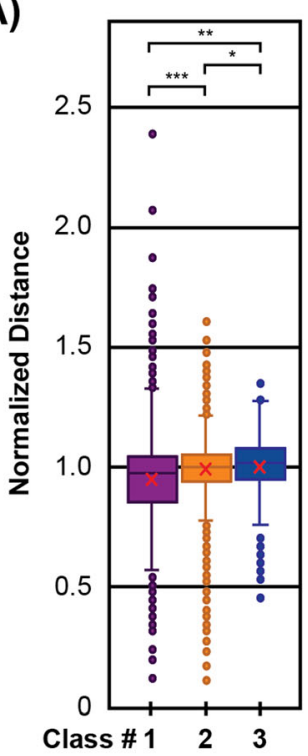

B)

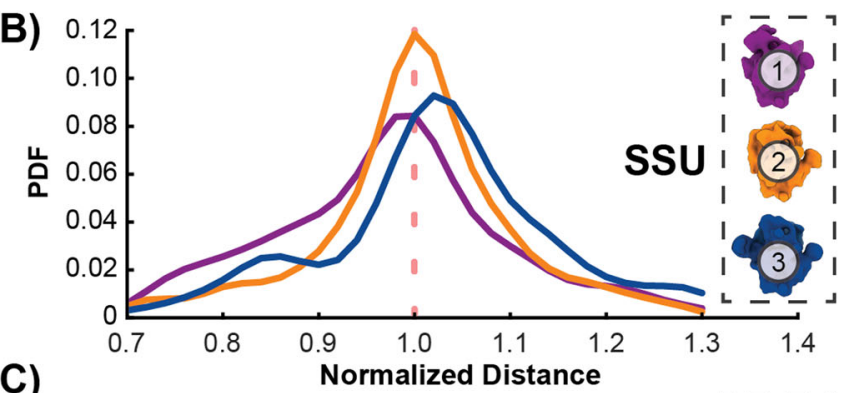

C)

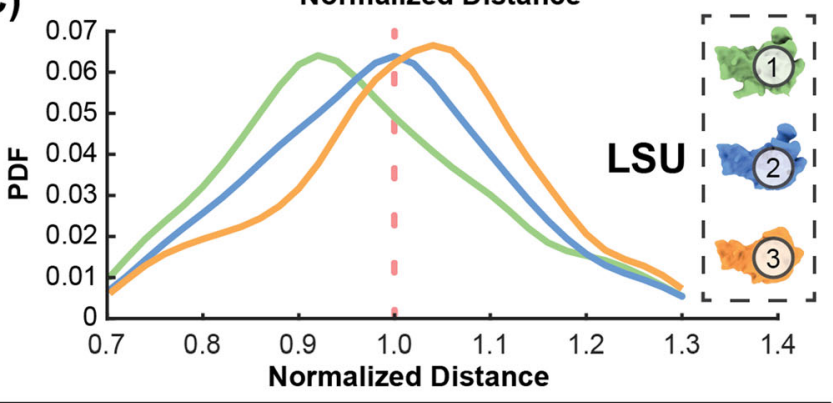

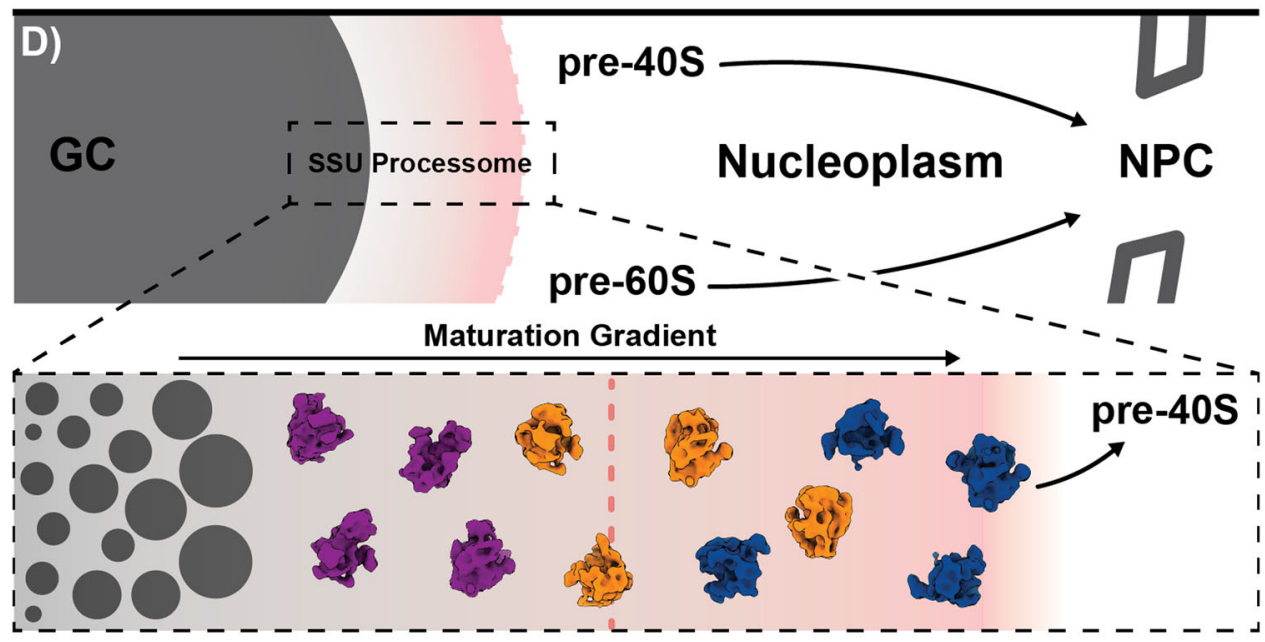

Fig. 4 Sub-Nucleolar Partitioning of Pre-Ribosomes. A The SSU processome classes show significant differences in their spatial distribution depending on their level of maturation ( $n=26$ tomograms; two-sided Fisher-Pitman permutation test 60 of mean: $p_{1,2}=1 \mathrm{E}-6, p_{1,3}=1.07 \mathrm{E}-04, p_{2,3}=4.1 \mathrm{E}-02$; $n=1809$ particles; data are represented as boxplots where the red cross indicates the mean, the middle line is the median, the lower and upper hinges correspond to the first and third quartiles, top and bottom whiskers indicate maximum and minimum, respectively. Circles show outlier points. See Supplementary Fig. 7d for pre-60S results. B, C Plotting the probability density function (PDF) for the large and small subunit precursors reveals discrete behaviors. There is a sharp peak for SSU Class $2 \& 3$ at the nucleolar surface and Class 1 displays higher probabilities at smaller radii. Likewise, pre-60S Class 1 shows a peak at even lower distances, implying that particles can still be detected further inside the nucleolus and that template matching is not restricted to its periphery ( $n=26$ tomograms). D The data suggests a model, in which pre-ribosomal particles are separated in maturation gradients and thus provide molecular order to the nucleolus and ribosome biogenesis. SSU Small subunit, LSU large subunit, GC Granular Component, NPC Nuclear Pore Complex. Source data are provided as a Source Data file.

cooled by an open nitrogen circuit. Lamellas were cut from randomly selected, mostly individual C. reinhardtii cells analogous to published protocols ${ }^{44}$

Cryo-TEM. Cryo-tomograms were acquired on a transmission electron microscope (Titan Krios, FEG $300 \mathrm{kV}$, FEI) with a post-column energy-filter (968 Quantum $\mathrm{K} 2$, Gatan) with a defocus range of $-5 \mu \mathrm{m}$ to $-3.5 \mu \mathrm{m}$ and an EFTEM magnification of $42000 x$ (calibrated pixel size $3.42 \AA$ ). Images were recorded with a direct detection camera (K2 Summit, Gatan) in dose-fractionation mode and a total dose of $\sim 120 \mathrm{e}^{-} / \AA^{2}$ per tomogram using SerialEM ${ }^{45}$. The acquisition was controlled by an in-house script running a dose-symmetric tilt scheme ${ }^{46}$ with an angle increment of $2^{\circ}$ between the range of $70^{\circ}$ and $-50^{\circ}$ starting at $10^{\circ}$ to compensate for the lamella pre-tilt $\left(\sim 12^{\circ}\right)$. In addition to the dose symmetric tilt schemes, 21 out of the total 85 tomograms were recorded using a bi-directional scheme with an angular increment of $2^{\circ}$ starting at $-20^{\circ}$ and going to $+70^{\circ}$. This was followed by a second branch from $-22^{\circ}$ to $-50^{\circ}$ to complete the series. Frames were aligned using motioncorr $2^{47}$, the contrast transfer function (CTF) estimated using CTFFIND4 48 , stacks were assembled using an in house Matlab (MathWorks) script, and tilt-series alignment as well as tomogram reconstruction was performed in IMOD ${ }^{49}$ using custom Matlab (MathWorks) wrapper scripts for batch pre-processing. To avoid bias, tomogram names were hashed to conceal the experimental state (log, stationary, DAZ-treated, synchronized-dark; see above).

Visualization of tomograms. To enhance the contrast of TEM insets, tomograms were denoised using TOPAZ ${ }^{50}$

\section{Bias-mitigated template matching, classification \& subtomogram averaging} To construct a featureless sphere, 300 nucleolar particles were manually picked and averaged in $\mathrm{PyTOM}^{51}$ with randomly assigned angles. Using this spherical reference, template matching was performed on binned (IMOD bin4, $13.68 \AA /$ pix) tomograms using PyTOM. The top 600 hits (as determined by a custom Matlab script) were extracted from each of the unbinned tomograms and subjected to an initial classification in Relion 2.1 ( $\mathrm{T}=4 ; 25$ iterations with CTF correction) allowing for up to 16 classes ${ }^{52,53}$. The two classes identified as SSU processome and LSU precursor were separated and refined individually, yielding the SSU and LSU precursor global averages depicted in Supplementary Fig. 2. 
Sphere fitting. From a selection of suitable tomograms where enough of the "nucleolar ring" was present (i.e. top or complete cuts; compare Supplementary Fig. 2), spheres were fit to the positions of the nucleolar SSU and LSU precursors using a custom Matlab (MathWorks) script. In brief, from an initial guess for center and radius (mean of all positions and mean distance to this center), a spherical equation was fit using a least square fit. Outlier particles $( \pm 1$ standard deviation by distance to the calculated center), were then removed and the fit was repeated. This procedure was repeated until no more changes were observed and the fit which the highest number of points retained was taken.

Density measurements. 2x binned (IMOD bin 4, $13.68 \AA$ /pixel) were denoised using neuronal networks $\left(\mathrm{TOPAZ}^{50}\right.$ ) and filtered using a Gaussian kernel (Matlab imgaussfilt3, sigma $=3$ pixel).

Denoised tomograms were normalized to a mean of zero and a standard deviation of one. The threshold for binarization was determined for every tomogram by measuring the mass of the densities at coordinates determined by template matching for the pre-ribosomal particles. The threshold was decrease until the mean mass of all detected subtomograms was $4.5 \mathrm{MDa}$, and then applied to the entire tomogram for binarization. Binarized volumes were segmented using Matlab's regionprops3, yielding xyz coordinates and the number of voxels for each object. For illustration, one such volume is shown in Fig. 2A (bottom), where densities were colored according to size in Chimera ${ }^{54}$. The corresponding mass of each subvolume was calculated based on voxel size and the protein density $\left(1.3 \mathrm{~g} \mathrm{~cm}^{-3}\right)$. Using aforementioned sphere fits, a distance to the center of the nucleolus was calculated for each $\mathrm{x}, \mathrm{y}, \mathrm{z}$ position. To make measurements comparable, all distances were then normalized by the radius of the determined spheres. Based on the mass and the normalized distance two types histograms were calculated:

One-dimensional histogram. Objects were grouped according to the distance and the mean mass was calculated for each bin (Fig. 2A).

Two-dimensional histogram. Objects were grouped according to mass and normalized distance. For each bin the protein density was computed (Fig. 2B).

Probability density functions. PDFs were calculated using Matlab's fitdist (normal kernel) function and pdf command.

Template matching using the truncation strategy and sub-classification. To ensure purely data-driven template matching, two truncated references were generated from the initial SSU processome structure (filtered to $40 \AA$ resolution; $13.68 \AA$ A $/$ pix) by computationally removing density using a spherical mask as indicated in Supplementary Fig. ${ }^{55}$. Two separate rounds of template matching were then performed in PyTOM $^{51}$ using these references. In order to over-pick, the 400 highest scoring hits were extracted from each tomogram and subjected to an initial classification using 40 classes at bin $4(13.68 \AA / \mathrm{pix})$ in Relion $2.1(\mathrm{~T}=4 ; 25$ iterations with CTF correction) to check for re-growth of the removed densities. Hits were retained if a particle had a corresponding point in both lists within \pm 4 -pixel distance of one another. This cleaned motive list was then used to extract particle sub-volumes from the twofold binned or unbinned tomograms (6.96 and $3.48 \AA$ Apix pixel, respectively). Subtomograms were first averaged and then subjected to 3D sub-classification with six classes in Relion 2.1 ( $\mathrm{T}=4 ; 25$ iterations with CTF correction), allowing for empty classes and yielding initial SSU processome class averages 1-3 after similarity-based tree clustering (Supplementary Fig. $5 \mathrm{C})^{56}$. Final relative class abundance and classification reproducibility was determined separately (see below). Differentiation into the individual experimental states (log, stationary, DAZ-treated, synchronized-dark; see above) was performed after resolving the tomogram names. Class occupancies are reported as percental fraction within the respective state. The LSU precursor was processed analogously.

Classification reproducibility. Subtomograms subjected to classification in Relion 2.1 were also classified using multireference alignment implemented in STOPGAP ${ }^{57}$. First, all subtomograms were aligned in stopgap starting with shifts and angles from Relion. There were no further improvements in FSC and map quality observed after two iterations of alignment. The resulting subtomogram alignment was used as a starting point for a multireference alignment. To determine the most variable parts of the structure, initial multireference classification was performed with a global mask. Starting references for the multireference alignment were generated by randomly assigning subtomograms to ten classes with an oversampling factor of two. Simulated annealing multireference alignment was performed against these starting references for ten iterations with decreasing temperature factors from 10 to 0 . The alignment was continued without simulated annealing for 30 iterations or until the class convergence $(<1 \%$ of subtomograms changing class during an iteration) was obtained. A stochastic hillclimbing search algorithm was used to score class assignments. This procedure, including generating random initial references was performed three times in total. The most variable region of a structure was determined after analyzing the resulting $30(10$ $\times 3)$ structures. Another round of simulated annealing multireference classification was performed with a mask focused on this region of the average using the procedure described above. However, for this step, we performed six replicates. Class occupancies after the class convergence for each replicate were observed to be similar (Supplementary Fig. 5A). The resulting ten structures from each of these six replicates were found to form three major clusters when subjected to hierarchical clustering (Supplementary Fig. 5B). Class consensus was defined as a subtomogram assignment to the same class, five out of six times. Final averages were generated using a consensus subtomogram assignment for each of the three classes.

Additionally, we checked for cross-effects, which might influence classification results. These include orientation, defocus, and lamella thickness (Supplementary Fig. 5D-F), none of which showed any significant correlation with class assignments.

Structural comparisons. EM maps from published structures (EMDB accession codes indicated in the figures) were re-scaled to the same pixel size as the STA averages and filtered to the same resolution $(25-35 \AA)$. After a global alignment using a fast rotational matching (FRM) algorithm, normalized cross-correlation coefficients were calculated in MatLab (MathWorks) from masked averages using custom scripts. For illustration and orientation in overviews, published molecular models were fit into the EM-densities using UCSF Chimera's molmap and fit-inmap commands at the respective STA map resolution.

Distance measurements. The distance of pre-ribosomes (LSU, SSU) were calculated with respect to the individual fit nucleolar centers (see 'Sphere Fitting') Distances are normalized with the respective mean nucleolar radius. The data was split into each individual class and treatment.

Data visualization. Membranes were segmented using membseg $2^{58}$ and manually improved using Amira (Thermo Fisher Scientific). Renderings and Movies of maps and models were produced in UCSF ChimeraX ${ }^{59}$.

Statistical and reproducibility. P-values were calculated using a Fisher-Pitman permutation test ${ }^{60}$. Significance is reported as follows: $P \geq 0.05$ : NS; $P \leq 0.05$ : *; $P \leq 0.01:{ }^{* *} ; P \leq 0.0001: * * *$.

Reporting summary. Further information on research design is available in the Nature Research Reporting Summary linked to this article.

\section{Data availability}

The data that support this study are available from the corresponding authors upon reasonable request. The subtomogram averages generated in this study were deposited in the EMDB database under accession numbers EMD-11046, 11047 and 11048 for the LSU Classes 1-3 and EMD-11043, 11044 and 11045 for the SSU Classes 1-3, respectively. Source data are provided with this paper.

\section{Code availability}

Any code used in this study is available from the authors upon request. An improved version of the pre-processing pipeline is now available on github as TOMOMAN [https://github.com/williamnwan/TOMOMAN.git].

Received: 20 February 2021; Accepted: 9 August 2021; Published online: 10 September 2021

\section{References}

1. Woolford, J. L. \& Baserga, S. J. Ribosome biogenesis in the yeast Saccharomyces cerevisiae. Genetics 195, 643-681 (2013).

2. Mougey, E. B. et al. The terminal balls characteristic of eukaryotic rRNA transcription units in chromatin spreads are rRNA processing complexes. Genes Dev. 7, 1609-1619 (1993).

3. Osheim, Y. N. et al. Pre-18S ribosomal RNA is structurally compacted into the SSU processome prior to being cleaved from nascent transcripts in Saccharomyces cerevisiae. Mol. Cell 16, 943-954 (2004).

4. Neyer, S. et al. Structure of RNA polymerase I transcribing ribosomal DNA genes. Nature 540, 607 (2016).

5. Boisvert, F.-M. M., van Koningsbruggen, S., Navascués, J. \& Lamond, A. I. The multifunctional nucleolus. Nat. Rev. Mol. Cell Biol. 8, 574-585 (2007).

6. Li, Z. F. \& Lam, Y. W. A new rapid method for isolating nucleoli BT. In The Nucleus (ed. Hancock, R.), Springer New York, New York, NY, 2015, pp. $35-42$.

7. Cheng, Y., Grigorieff, N., Penczek, P. A. \& Walz, T. A primer to single-particle cryo-electron microscopy. Cell 161, 439-449 (2015).

8. Kornprobst, M. et al. Architecture of the 90S pre-ribosome: a structural view on the birth of the eukaryotic ribosome. Cell 166, 380-393 (2016).

9. Cheng, J., Kellner, N., Berninghausen, O., Hurt, E., \& Beckmann, R., 3. 2- $\AA$ resolution structure of the $90 \mathrm{~S}$ preribosome before A1 pre-rRNA cleavage. Nat. Struct. Mol. Biol., 24, 954-964 (2017). 
10. Sun, Q. et al. Molecular architecture of the $90 \mathrm{~S}$ small subunit pre-ribosome Elife 1, 1-28 (2017).

11. Sanghai, Z. A. et al. Modular assembly of the nucleolar pre-60S ribosomal subunit. Nature 556, 126-129 (2018).

12. Kater, L. et al. Visualizing the assembly pathway of nucleolar pre-60S ribosomes. Cell 171, 1599-1610.e13 (2017).

13. Johnson, M. C., Ghalei, H., Doxtader, K. A., Karbstein, K. \& Stroupe, M. E. Structural heterogeneity in pre-40S ribosomes. Structure 25, 329-340 (2017).

14. Hunziker, M. et al. Conformational switches control early maturation of the eukaryotic small ribosomal subunit. Elife 8, 1-16 (2019).

15. Wu, S. et al. Diverse roles of assembly factors revealed by structures of late nuclear pre-60S ribosomes. Nature 534, 133-137 (2016).

16. Bradatsch, B. et al. Structure of the pre-60S ribosomal subunit with nuclear export factor Arx1 bound at the exit tunnel. Nat. Struct. Mol. Biol. 19, 1234-1241 (2012).

17. Beck, M. \& Baumeister, W. Cryo-electron tomography: can it reveal the molecular sociology of cells in atomic detail? Trends Cell Biol. 26, 825-837 (2016).

18. Förster, F., Han, B.-G., \& Beck, M., Chapter Eleven - visual proteomics. In Cryo-EM, part C: analyses, interpretation, and case studies (ed. Jensen, G. J.), Academic Press, 2010, pp. 215-243.

19. Schaffer, M. et al. Cryo-focused ion beam sample preparation for imaging vitreous cells by cryo-electron tomography. Bio Protoc. 5, e1575 (2015).

20. Plitzko, M., Villa, E., Schaffer, M. \& Baumeister, W. Opening windows into the cell: focused-ion-beam milling for cryo-electron tomography. Curr. Opin. Struct. Biol. 23, 771-777 (2013).

21. Hanson, P. K. Saccharomyces cerevisiae: a unicellular model genetic organism of enduring importance. Curr. Protoc. Essent. Lab. Tech. 16, 1-15 (2018).

22. Yang, C. H., Lambie, E. J., Hardin, J., Craft, J. \& Snyder, M. Higher order structure is present in the yeast nucleus: autoantibody probes demonstrate that the nucleolus lies opposite the spindle pole body. Chromosoma 98, 123-128 (1989).

23. Kim, S. et al. The dynamic three-dimensional organization of the diploid yeast genome. Elife 6, 1-23 (2017).

24. Arnold, J. et al. Site-specific cryo-focused ion beam sample preparation guided by 3D correlative microscopy. Biophys. J. 110, 860-869 (2016).

25. Ebersberger, I. et al. The evolution of the ribosome biogenesis pathway from a yeast perspective. Nucleic Acids Res. 42, 1509-1523 (2014).

26. Of, D. \& Chloroplast, T. H. E. Structure and development of the chloroplast in chlamydomonas. J. Biophys. Biochem Cytol. 3, 463-488 (1957).

27. Böhm, J. et al. Toward detecting and identifying macromolecules in a cellular context: Template matching applied to electron tomograms. Proc. Natl Acad. Sci. USA 97, 14245 LP-14214250 (2000).

28. Frangakis, A. S. et al. Identification of macromolecular complexes in cryoelectron tomograms of phantom cells. Proc. Natl Acad. Sci. USA 99, 14153-14158 (2002).

29. Klinge, S. \& Woolford, J. L. Ribosome assembly coming into focus. Nat. Rev. Mol. Cell Biol. 20, 116-131 (2019).

30. Inwood, W., Yoshihara, C., Zalpuri, R., Kim, K. S. \& Kustu, S. The ultrastructure of a chlamydomonas reinhardtii mutant strain lacking phytoene synthase resembles that of a colorless alga. Mol. Plant 1, 925-937 (2008).

31. Trumtel, S., Léger-Silvestre, I., Gleizes, P. E., Teulières, F. \& Gas, N. Assembly and functional organization of the nucleolus: Ultrastructural analysis of Saccharomyces cerevisiae mutants. Mol. Biol. Cell 11, 2175-2189 (2000).

32. Yao, R.-W. W. et al. Nascent pre-rRNA sorting via phase separation drives the assembly of dense fibrillar components in the human nucleolus. Mol. Cell 76, 767-783.e11 (2019).

33. Feric, M. et al. Coexisting liquid phases underlie nucleolar subcompartments. Cell 165, 1686-1697 (2016).

34. Riback, J. A. et al. Composition-dependent thermodynamics of intracellular phase separation. Nature 581, 209-214 (2020).

35. Schaffer, M. et al. A cryo-FIB lift-out technique enables molecular-resolution cryo-ET within native Caenorhabditis elegans tissue. Nat. Methods 16, 757-762 (2019).

36. $\mathrm{Du}, \mathrm{Y}$. et al. Cryo-EM structure of $90 \mathrm{~S}$ small ribosomal subunit precursors in transition states. Science 369, 1477-1481 (2020).

37. Lau, B. et al. Structure of the maturing $90 \mathrm{~S}$ pre-ribosome in association with the RNA exosome. Mol. Cell, 81, 293-303.e4 (2021).

38. Barandun, J. et al. The complete structure of the small-subunit processome. Nat. Struct. Mol. Biol., 24, 944-953 (2017).

39. Loibl, M. et al. The drug diazaborine blocks ribosome biogenesis by inhibiting the AAA-ATPase drg1. J. Biol. Chem. 289, 3913-3922 (2014).

40. Kawashima, S. A. et al. Potent, reversible, and specific chemical inhibitors of eukaryotic ribosome biogenesis. Cell 167, 512-524.e14 (2016).

41. Zones, J. M., Blaby, I. K., Merchant, S. S. \& Umen, J. G. High-resolution profiling of a synchronized diurnal transcriptome from Chlamydomonas reinhardtii reveals continuous cell and metabolic differentiation. Plant Cell 27, 2743-2769 (2015).
42. Weis, B. L., Kovacevic, J., Missbach, S. \& Schleiff, E. Plant-specific features of ribosome biogenesis. Trends Plant Sci. 20, 729-740 (2015)

43. Umen, J. G. \& Goodenough, U. W. Control of cell division by a retinoblastoma protein homolog in Chlamydomonas. Genes Dev. 15, 1652-1661 (2001).

44. Schaffer, M. et al. Optimized cryo-focused ion beam sample preparation aimed at in situ structural studies of membrane proteins. J. Struct. Biol. 197, 73-82 (2017)

45. Mastronarde, D. N. Automated electron microscope tomography using robust prediction of specimen movements. J. Struct. Biol. 152, 36-51 (2005).

46. Hagen, W. J. H., Wan, W. \& Briggs, J. A. G. Implementation of a cryo-electron tomography tilt-scheme optimized for high resolution subtomogram averaging. J. Struct. Biol. 197, 191-198 (2017).

47. Zheng, S. Q. et al. MotionCor2: anisotropic correction of beam-induced motion for improved cryo-electron microscopy. Nat. Methods 14, 331-332 (2017).

48. Rohou, A. \& Grigorieff, N. CTFFIND4: fast and accurate defocus estimation from electron micrographs. J. Struct. Biol. 192, 216-221 (2015).

49. Kremer, J. R., Mastronarde, D. N. \& McIntosh, J. R. Computer visualization of three-dimensional image data using IMOD. J. Struct. Biol. 116, 71-76 (1996).

50. Bepler, T., Kelley, K., Noble, A. J. \& Berger, B. Topaz-Denoise: general deep denoising models for cryoEM and cryoET. Nat. Commun. 11, 1-12 (2020).

51. Hrabe, T. et al. PyTom: a python-based toolbox for localization of macromolecules in cryo-electron tomograms and subtomogram analysis. $J$. Struct. Biol. 178, 177-188 (2012).

52. Bharat, T. A. M. \& Scheres, S. H. W. Resolving macromolecular structures from electron cryo-tomography data using subtomogram averaging in RELION. Nat. Protoc. 11, 2054-2065 (2016).

53. Fernandez-Leiro, R. \& Scheres, S. H. W. A pipeline approach to single-particle processing in RELION. Acta Crystallogr. Sect. D. Struct. Biol. 73, 496-502 (2017).

54. Pettersen, E. F. et al. UCSF Chimera - a visualization system for exploratory research and analysis. J. Comput. Chem. 25, 1605-1612 (2004).

55. Yu, Z. \& Frangakis, A. S. M-free: scoring the reference bias in sub-tomogram averaging and template matching. J. Struct. Biol. 187, 10-19 (2014).

56. Förster, F. \& Hegerl, R. Structure determination in situ by averaging of tomograms. Methods Cell Biol. 2007, 741-767 (2007).

57. Wan, W., GitHub https://github.com/williamnwan/STOPGAP/tree/0.7.1 (2020).

58. Martinez-Sanchez, A., Garcia, I., Asano, S., Lucic, V. \& Fernandez, J.-J. Robust membrane detection based on tensor voting for electron tomography. J. Struct. Biol. 186, 49-61 (2014).

59. Goddard, T. D. et al. UCSF ChimeraX: meeting modern challenges in visualization and analysis. Protein Sci. 27, 14-25 (2018).

60. Neuhäuser, M. \& Manly, B. F. J. The Fisher-Pitman permutation test when testing for differences in mean and variance. Psychol. Rep. 94, 189-194 (2004).

\section{Acknowledgements}

We thank B. Engel and W. Wietrzynski for discussions and O. Schiøtz for critical reading of the manuscript. We thank VE for help preparing the figures and IW for supporting the IT infrastructure. We acknowledge support by an Alexander von Humboldt returners fellowship to P.S.E. and an EMBO Long-term Fellowship ALTF 764-2014 to F.W.

\section{Author contributions}

Conceptualization: P.S.E., W.B. Methodology: P.S.E., F.B., J.M.P., W.B. Investigation: P.S.E., Z.H., Sa.K., S.K., F.W. Visualization: P.S.E., F.B. Funding Acquisition: P.S.E., J.M.P., W.B. Project Administration, P.S.E., W.B. Supervision: P.S.E., W.B. Writing - original draft: P.S.E., W.B. Writing - review \& editing: P.S.E., Z.H., Sa.K., S.K., F.B., J.M.P., W.B.

\section{Funding}

Open Access funding enabled and organized by Projekt DEAL.

\section{Competing interests}

The authors declare no competing interests.

\section{Additional information}

Supplementary information The online version contains supplementary material available at https://doi.org/10.1038/s41467-021-25413-w.

Correspondence and requests for materials should be addressed to P.S.E. or W.B.

Peer review information Nature Communications thanks the anonymous reviewers for their contribution to the peer review of this work. Peer reviewer reports are available.

Reprints and permission information is available at http://www.nature.com/reprints

Publisher's note Springer Nature remains neutral with regard to jurisdictional claims in published maps and institutional affiliations. 
(c) (i) Open Access This article is licensed under a Creative Commons Attribution 4.0 International License, which permits use, sharing, adaptation, distribution and reproduction in any medium or format, as long as you give appropriate credit to the original author(s) and the source, provide a link to the Creative Commons license, and indicate if changes were made. The images or other third party material in this article are included in the article's Creative Commons license, unless indicated otherwise in a credit line to the material. If material is not included in the article's Creative Commons license and your intended use is not permitted by statutory regulation or exceeds the permitted use, you will need to obtain permission directly from the copyright holder. To view a copy of this license, visit http://creativecommons.org/ licenses/by/4.0/.

(C) The Author(s) 2021 